Revista de Comunicación y Salud, 2019, Vol. 9, n 1, pp. 19-38

Editado por Cátedra de Comunicación y Salud

ISSN: 2173-1675

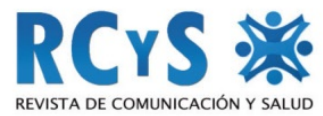

Enviado 16/05/2019

Aprobado 10/06/2019

\title{
NECESIDAD DE FORMAR A LOS CUIDADORES DE PERSONAS EN SITUACIÓN DE DEPENDENCIA: COMUNICACIÓN Y RESPUESTA DESDE LA UCM
}

Need to train caregivers of people in situations of dependency: communication and response from the UCM

Miguel Ángel Carretero Díaz ${ }^{1}$. Universidad Complutense de Madrid. España. miguelangelcarretero@ucm.es

Almudena Barrientos-Báez. Escuela Universitaria de Turismo Iriarte. España. almudenabarrientos@iriarteuniversidad.es

\section{Resumen}

Recientemente se está incrementando la necesidad de proporcionar cuidados a las personas con diverso grado de dependencia y vulnerabilidad, que cada vez viven más años debido fundamentalmente a dos razones: los avances científicos en el ámbito de la salud y los cambios familiares, laborales y sociales. Estos cuidados suelen ser impartidos por familiares -principalmente mujeres- que deben ocuparse durante las 24 horas del día en la soledad del domicilio de estas personas, sin hablar, sin comunicarse y muchas veces sin recibir un feedback que anime a seguir. Se trata de una tarea difícil y prolongada, sin apenas reconocimiento, aunque también pueda despertar facetas insospechadas que enriquezcan al cuidador. Lógicamente estos realizan su inestimable labor con más voluntad, interés y dedicación que conocimientos, porque nadie les ha indicado claramente cómo debe realizarla. En consecuencia, desde una universidad pública como la Universidad Complutense de Madrid, una institución educativa con una clara vocación de servicio surge una oferta formativa para estos cuidadores con el claro objetivo de paliar esta necesidad creciente.

Palabras clave: comunicación; cuidadores; dependencia; emoción, formación.

\section{Abstract}

Recently, the need to provide care for people with different degrees of dependency and vulnerability is growing, and they are living longer, mainly due to two reasons: scientific advances in the field of health and family, work and social changes. This care is usually

1 Autor para correspondencia: Miguel Ángel Carretero Díaz miquelangelcarretero@ucm.es 
Necesidad de formar a los cuidadores de personas en situación de dependencia: comunicación y respuesta desde la UCM

given by family members -mainly women- who must work 24 hours a day in the solitude of their homes, without speaking, without communicating and often without receiving feedback that encourages them to follow. It is a difficult and prolonged task, with little recognition, although it can also awaken unsuspected facets that enrich the caregiver. Logically they perform their invaluable work with more will, interest and dedication than knowledge, because no one has clearly indicated how to perform it. Consequently, from a public university such as the Complutense University of Madrid, an educational institution with a clear vocation for service, a training offer for these caregivers emerges with the clear objective of alleviating this growing need.

Keywords: communication; caregivers; dependence; emotion, training.

\section{Cómo citar el artículo}

Carretero Díaz, M. A. y Barrientos-Báez, A. (2019). Necesidad de formar a los cuidadores de personas en situación de dependencia: comunicación y respuesta desde la UCM. Revista de Comunicación y Salud, 9(1), 19-38.

doi: http://doi.org/10.35669/revistadecomunicacionysalud.2019.9(1).19-38

\section{INTRODUCCIÓN}

La necesidad de proporcionar cuidados de calidad a una población dependiente cada vez más numerosa obliga a las organizaciones educativas a responder desde un punto de vista formativo, planificando y ofreciendo una oferta curricular apropiada para que los denominados "cuidadores no profesionales» puedan comunicar y desarrollar sus funciones de la mejor manera posible a estas personas en situación de dependencia y vulnerabilidad, temporal o permanente. Se trata de ofrecer pues una respuesta a las necesidades crecientes de formación de los cuidadores pero también de sintonizar con la aplicación de la Ley 39/2006 de 14 de diciembre, de Promoción de la Autonomía Personal y Atención a las personas en situación de dependencia favoreciendo su desarrollo.

\section{ANTECEDENTES Y JUSTIFICACIÓN}

Los cambios demográficos y sociológicos acaecidos durante estas últimas décadas en la población española están provocando nuevas y crecientes necesidades en la asistencia y el cuidado de una enorme cantidad de personas en situación de vulnerabilidad y dependencia y en consecuencia grandes dificultades para su desenvolvimiento vital.

Por otra parte, los avances científicos acaecidos han provocado altas tasas de población que han logrado cronificar la morbilidad o discapacidad de estas personas aquejadas de enfermedades tales como el cáncer o el SIDA, así como altas tasas de envejecimiento en la población, lo que se ha denominado como "el envejecimiento del envejecimiento". Todo ello está provocando el auge de la presencia -en muchos casos 
Necesidad de formar a los cuidadores de personas en situación de dependencia:

comunicación y respuesta desde la UCM

forzosa- de «cuidadores no profesionales», familiares cercanos o personas contratadas, con más entusiasmo que conocimientos, con más actitud que aptitud, que prestan dichos cuidados sin la preparación adecuada para ofrecer a estas personas dependientes un servicio acorde a sus necesidades.

Estos cambios científicos, demográficos y sociológicos demandan por una parte la necesidad de garantizar una estabilidad de recursos y servicios y, por lo tanto, el incremento y actualización de los servicios sociales y sanitarios, así como las imprescindibles modificaciones en el comportamiento familiar, y por otra, ofrecer un incremento de la oferta formativa adecuada para la figura de estos "cuidadores no profesionales", con el objetivo de puedan ofrecer una atención eficiente y de esta forma asegurar cuidados esenciales a estas personas en situación de dependencia o vulnerabilidad.

\subsection{El auge en la necesidad de proporcionar cuidados. La situación en España}

La Encuesta Nacional de Salud de España (ENSE) 2017 con respecto a la necesidad de proporcionar cuidados, refleja ${ }^{2}$ :

- El envejecimiento de la población residente en España. (2018, p.1)

- El aumento de la prevalencia de enfermedades crónicas, los factores de riesgo cardiovascular metabólicos (diabetes, hipertensión, hipercolesterolemia, obesidad...), y las enfermedades del aparato locomotor (artrosis, dolor lumbar), y también se incrementa la población con limitaciones y discapacidad funcional. $(2018$, p. 1)

- La limitación de la movilidad es la principal causa de discapacidad. En mayores de 64 años afecta al 45,3\%. En este mismo grupo de edad, el $44,1 \%$ refirió dificultades auditivas, algo por encima del $41,7 \%$ de 2014 , y el $24,3 \%$ dificultades visuales, semejante a años anteriores. Por primera vez se explora la dificultad cognitiva, que alcanza al $31,3 \%$ de la población de 65 y más años. $(2018$, p. 5).

${ }^{2}$ Encuesta Nacional de Salud. España 2017.

https://www.ine.es/dyngs/INEbase/es/operacion.htm?c=Estadistica C\&cid=1254736176783\&menu=result ados\&idp=1254735573175 (Consultado 15-02-2019).

Nota Técnica. (2018) Ministerio de Sanidad, Consumo y Bienestar Social. Gobierno de España. https://www.mscbs.gob.es/estadEstudios/estadisticas/encuestaNacional/encuestaNac2017/ENSE2017 n otatecnica.pdf (Consultado 15-02-2019).

Revista de Comunicación y Salud, 2019, Vol. 9, n 1, pp. 19-38 
Necesidad de formar a los cuidadores de personas en situación de dependencia:

comunicación y respuesta desde la UCM
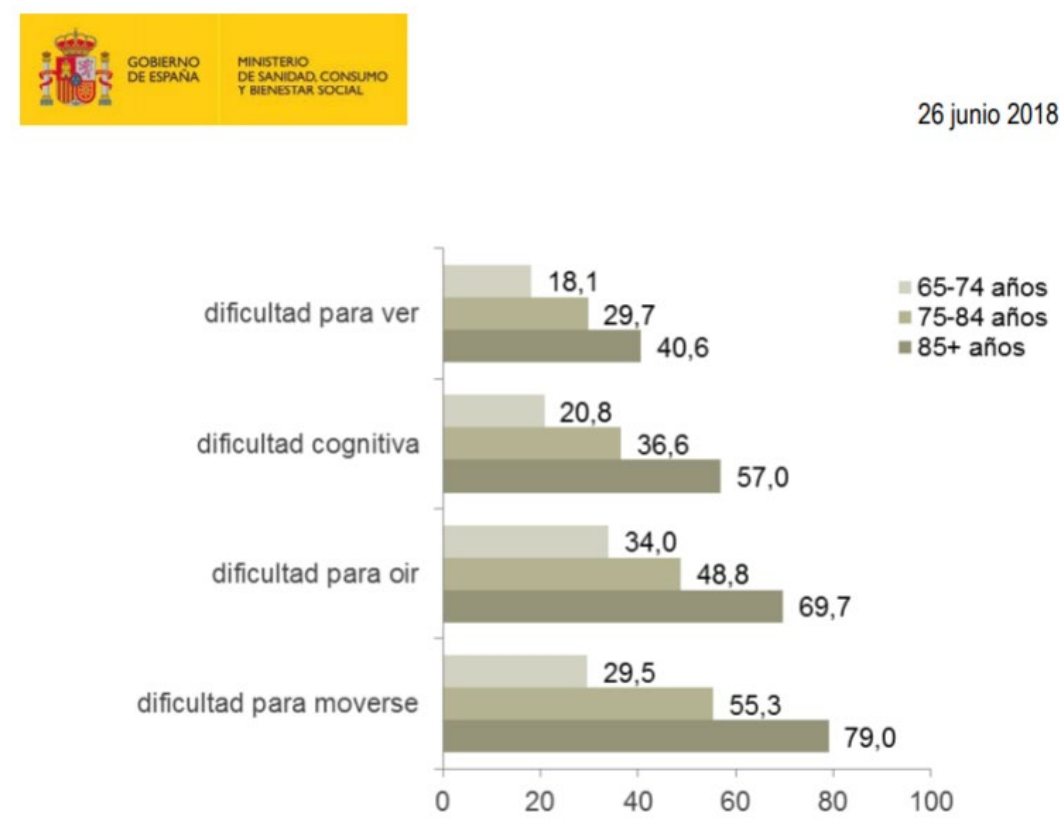

Figura 1

- Dos de cada diez mayores (19,6\%) refiere algún grado de dificultad para alguna actividad de cuidado personal (alimentarse, sentarse y levantarse, vestirse, ir al servicio, ducharse), con mayor frecuencia las mujeres $(24,5 \%)$ que los hombres $(13,2 \%)$

- De las personas con dificultad, el $84,9 \%$ dispone de ayudas técnicas o personales al menos para una de las actividades, pero el $57,7 \%$ necesita ayuda o más ayuda de la que dispone.

- En el caso de las actividades domésticas (preparar la comida, utilizar el teléfono, realizar compras, tomar medicamentos, tareas domésticas ligeras y pesadas, administrar el dinero), una de cada dos personas de 65 y más años $(52,4 \%)$, presenta algún grado de dificultad para alguna de ellas, con mayor frecuencia las mujeres $(64,1 \%)$ que los hombres $(37,3 \%)$. De ellas, el $80,4 \%$ dispone de ayuda técnica o personal para realizar al menos una de las actividades y el $43,4 \%$ necesita ayuda o más ayuda de la que dispone. (2018, p. 6).

\subsection{El auge en la necesidad de proporcionar cuidados. La situación en el mundo}

Según el Informe Mundial sobre la Discapacidad (2011:7-8)

- Se estima que más de mil millones de personas viven con algún tipo de discapacidad; o sea, alrededor del $15 \%$ de la población mundial (según las estimaciones de Resumen Informe mundial sobre la discapacidad 8 la población mundial en 2010).

\footnotetext{
${ }^{3}$ https://www.who.int/disabilities/world report/2011/summary es.pdf?ua=1 (Consultado 15-02-2019)
} 
Necesidad de formar a los cuidadores de personas en situación de dependencia:

comunicación y respuesta desde la UCM

- Según la Encuesta Mundial de Salud, cerca de 785 millones de personas $(15,6 \%)$ de 15 años y más viven con una discapacidad, mientras que el proyecto sobre la Carga Mundial de Morbilidad estima una cifra próxima a los 975 millones $(19,4 \%)$.

- La Encuesta Mundial de Salud señala que, del total estimado de personas con discapacidad, 110 millones $(2,2 \%)$ tienen dificultades muy significativas de funcionamiento, mientras que la Carga Mundial de Morbilidad cifra en 190 millones $(3,8 \%)$ las personas con una "discapacidad grave" (el equivalente a la discapacidad asociada a afecciones tales como la tetraplejía, depresión grave o ceguera).

- Solo la Carga Mundial de Morbilidad mide las discapacidades infantiles (0-14 años), con una estimación de 95 millones de niños $(5,1 \%), 13$ millones de los cuales $(0,7 \%)$ tienen "discapacidad grave".

\subsection{Los resultados de la Encuesta de Discapacidad, Autonomía personal y situaciones de Dependencia (EDAD)}

Los datos de los que se dispone en España por parte del Instituto Nacional de Estadística corresponden -a falta de la prometida actualización de 2017-, a los correspondientes a la Encuesta de Discapacidad, Autonomía personal y situaciones de Dependencia (EDAD) del año 2008.

En 2008 hay residentes en hogares que afirman tener discapacidad o limitación ${ }^{4}$. Esto supone una tasa de habitantes El estudio de las características de la discapacidad se ha centrado en la población de 6 o más años, ya que para los menores el pronóstico de evolución es incierto y solo se analizan las limitaciones adaptadas a su edad. Para las personas de 6 o más años la tasa de discapacidad se sitúa en 89,7 por mil habitantes. 3,85 millones de personas 85,5 por mil.

\footnotetext{
${ }^{4}$ A efectos de la encuesta, se ha definido como la limitación por motivos de salud y de larga duración en alguna de las 44 actividades investigadas. Las personas con discapacidad se ven limitadas a causa de una deficiencia corporal (fallo o falta de un órgano o sistema).
} 
Necesidad de formar a los cuidadores de personas en situación de dependencia: comunicación y respuesta desde la UCM

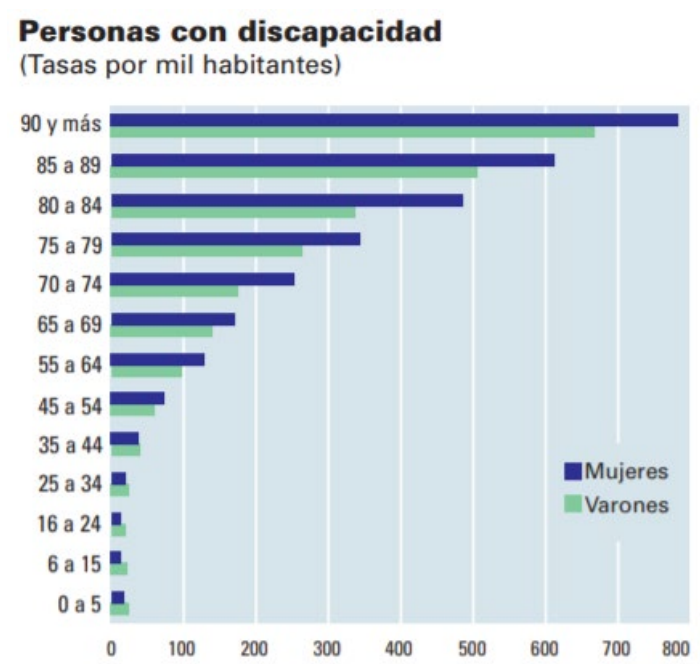

\begin{tabular}{|c|c|c|}
\hline \multirow[b]{3}{*}{ Total } & \multicolumn{2}{|c|}{ Tasas por mil hab. } \\
\hline & Varones & Mujeres \\
\hline & 72,6 & 106,3 \\
\hline Movilidad & $\overline{42,6}$ & 77,5 \\
\hline Vida doméstica & 29,5 & 69,2 \\
\hline Autocuidado & 31,3 & 55,3 \\
\hline Audición & 21,9 & 28,4 \\
\hline Visión & 17,8 & 28,4 \\
\hline Comunicación & 16,3 & 18,6 \\
\hline $\begin{array}{l}\text { Aprendizaje y aplicación de } \\
\text { conocimientos y desarrollo de tareas }\end{array}$ & 12,7 & 17,1 \\
\hline Interacciones y relaciones personales & 14,0 & 15,4 \\
\hline
\end{tabular}

\section{Figura 2}

La movilidad, la más habitual: el $67,2 \%$ de estas personas presentan limitaciones para moverse o trasladar objetos, el $55,3 \%$ tienen problemas relacionados con las tareas domésticas y el $48,4 \%$ con las tareas del cuidado e higiene personal.

¿Qué causa más discapacidad?: la deficiencia más frecuente es la osteoarticular: debido a un problema en huesos y articulaciones, el $42,0 \%$ de las personas tiene discapacidad. Pero la deficiencia que causa mayor número de discapacidades por persona es la mental: 11,6 frente a las 8,7 de media que tienen las personas con discapacidad.

Con respecto a los diferentes tipos de discapacidades, destacan las que impiden realizar las Actividades Básicas de la Vida Diaria (ABVD) porque generan dependencia:

El número de estas personas alcanza los 2,8 millones y supone el $6,7 \%$ de la población (2008, p. 3).

\begin{tabular}{|c|c|c|c|c|c|c|}
\hline \multirow[b]{3}{*}{ Total } & \multirow[b]{2}{*}{ Total } & \multicolumn{5}{|c|}{ Número de discapacidades (ABVD) de la persona } \\
\hline & & $1-2$ & 3-5 & 6-9 & $10-13$ & 14-18 \\
\hline & $2.822,3$ & 861,3 & 761,4 & 563,6 & 341,5 & 294,4 \\
\hline \multicolumn{7}{|l|}{ Discapacidad } \\
\hline Moderada & 560,8 & 387,5 & 136,7 & 31,4 & 4,6 & 0,7 \\
\hline Severa & 702,2 & 258,0 & 264,9 & 130,7 & 37,1 & 11,5 \\
\hline Total & $1.383,6$ & 142,8 & 314,4 & 371,0 & 284,5 & 270,8 \\
\hline No consta & 175,7 & 73,0 & 45,4 & 30,5 & 15,4 & 11,4 \\
\hline
\end{tabular}

\section{Figura 3}


Necesidad de formar a los cuidadores de personas en situación de dependencia: comunicación y respuesta desde la UCM

\subsection{El perfil de los “cuidadores no profesionales" en España}

Corresponde al de una mujer que une a las tareas del trabajo doméstico, las tareas de cuidar de algún familiar cercano: padre, hermano, marido o hijo, lo que tiende a provocarles una sobrecarga tanto física como emocional provocada por dos factores sobresalientes: la necesaria dedicación continuada al cuidado y su falta de información y formación para realizar de una manera segura y eficiente su tarea como "cuidador no profesional".

También está emergiendo la figura creciente del "cuidador no profesional» contratado por los familiares que no pueden hacerse cargo de la persona en situación de vulnerabilidad y dependencia. Se trata de cuidadores sin formación alguna, muchos de ellos de origen suramericano o de los Países del Este: Rumanía, Bulgaria o Bielorrusia, recién venidos a España, que en su mayoría desconocen las básicas normas de higiene, prevención y las tareas principales del cuidado.

Tanto un tipo como otro de "cuidador no profesional" realizan su labor de manera continuada, en unos horarios que en la mayoría de los casos suelen resultar excesivos, sin reconocimiento salarial adecuado y con una tremenda sensación de soledad y de reconocimiento social por su labor, (Síndrome Burnout como el ilustrado en cuidadores de pacientes de Alzheimer por Martínez Pérez, 2010) por lo que suele resultar frecuente que acaben sufriendo somatización de enfermedades, trastornos del sueño 0 desequilibrios psicológicos que les conducen a estados depresivos.

En la Encuesta Nacional de Salud España (2017) se refiere:

Los cuidadores informales de personas mayores con problemas de salud o con alguna dolencia crónica proporcionan información indirecta de posibles carencias de servicios de cuidados de larga duración. Según los datos de la ENSE 2017, se mantienen estables con respecto a 2014 . El 11,2\% de los entrevistados declaró prestar cuidados al menos una vez a la semana, con mayor frecuencia las mujeres $(12,9 \%)$ que los hombres $(9,5 \%)$. Entre los 45 y los 64 años es cuando se prestan cuidados a personas mayores o enfermas con mayor frecuencia. El 27,1\% de las personas cuidadoras informales de clase I declara que dedica 20 o más horas semanales, frente al $55 \%$ de clase VI. (Encuesta Nacional de Salud España, 2018, p. 6).

La Encuesta de Discapacidad, Autonomía personal y situaciones de Dependencia refiere que:

Más de dos millones de personas con discapacidad reciben cuidados personales o supervisión. Estas tareas son prestadas por personas de su entorno o contratadas para tal fin.

Tres de cada cuatro cuidadores principales son mujeres. El perfil del cuidador principal es una mujer, de entre 45 y 64 años, que reside en el mismo hogar que la persona a la que presta cuidados.

Revista de Comunicación y Salud, 2019, Vol. 9, nº 1, pp. 19-38 
Necesidad de formar a los cuidadores de personas en situación de dependencia: comunicación y respuesta desde la UCM

Problemas de los cuidadores principales. Las personas que prestan cuidados sienten dificultades para desempeñar sus tareas. Además, se ven afectadas en su salud y vida personal. Dentro de las tareas de asistencia personal, la principal dificultad que encuentran los cuidadores es la falta de fuerza física para realizarlas. En cuanto a las consecuencias para su salud, la mayoría de ellos afirma sentirse cansada y una parte importante ve incluso deteriorada su salud. (La Encuesta de Discapacidad, Autonomía personal y situaciones de Dependencia, 2008, p. 4).
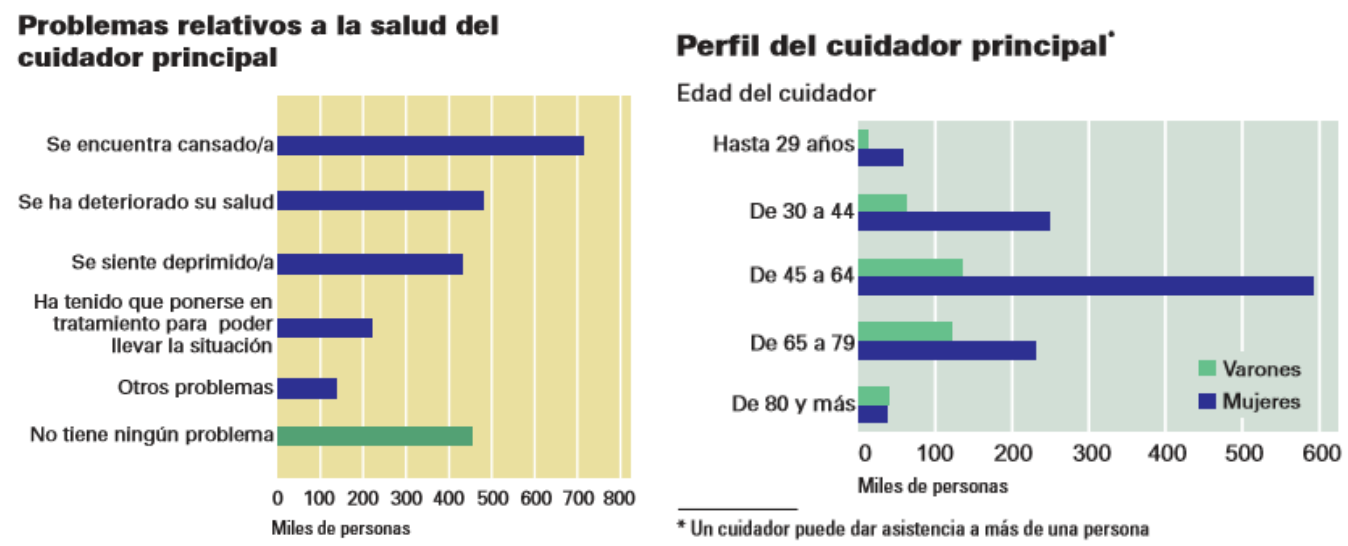

Figura 4

Para Artaza Artabe, I. y Ramos Cordero, P.:

1) El perfil del cuidador podría venir definido por:

a) Mujer $(88,5 \%)$

b) Entre 31 y 60 años (82,5\%)

c) Con estudios secundarios o superiores (88 \%)

d) Que compagina cuidados y trabajo $(63,5 \%)$

2) El cuidador tiende a la profesionalización:

a) Lleva más de 3 años cuidando (70 \%)

b) Dedica más de 6 horas/día (62\%)

c) Su trabajo es predominantemente urbano (68 \%)

3) El cuidador mayoritariamente:

a) No tiene parentesco $(52,5 \%)$

b) No vive con la persona cuidada (68\%)

c) Presta los cuidados en el domicilio de la persona a la que cuida mayoritariamente $(87 \%)$

d) Recibe ayuda complementaria (69 \%) 
Necesidad de formar a los cuidadores de personas en situación de dependencia: comunicación y respuesta desde la UCM
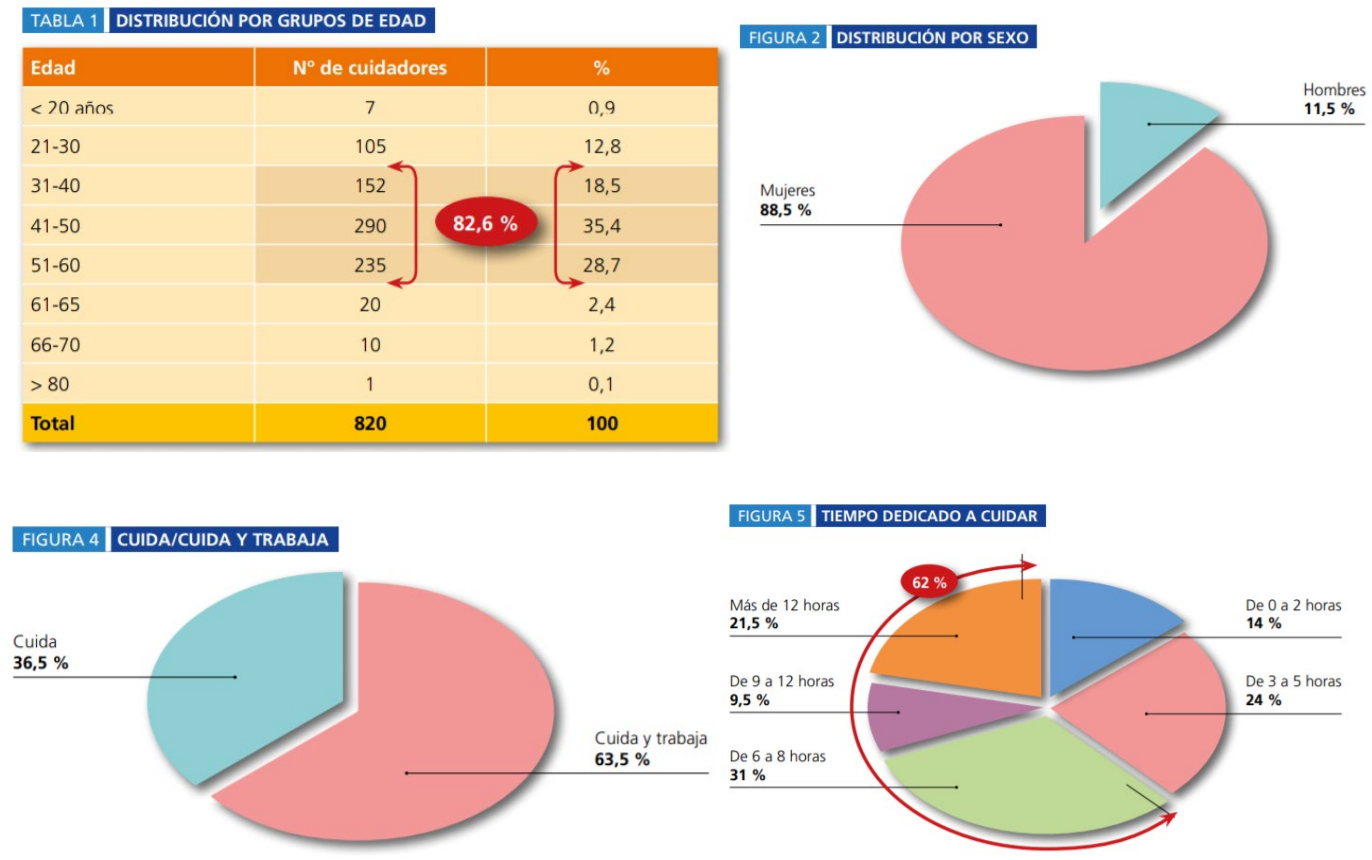

Figura 5

Estos "cuidadores no profesionales", a pesar de sus dificultades en el acceso a la información y a la formación para desarrollar de manera cotidiana sus importantes tareas de cuidado personal, deben afrontar decisiones y actuaciones cotidianas con gran desconocimiento de las posibles consecuencias en la persona cuidada. Además, emerge una necesidad para el cuidador y el "cuidado", y ésta hace referencia a la manera de comunicarse. Nos planteamos la necesidad de formar a los cuidadores en comunicación y técnicas del lenguaje verbal y no verbal porque en muchas ocasiones un simple gesto en la persona que se cuida es más valorado que pasar un paño limpio por la barbilla del paciente después de comer. Se trata de un ejemplo muy significativo porque además de cuidar, se debería emocionar: la aplicación de inteligencia emocional con el fin de alcanzar el bienestar psicológico (Suberviola-Ovejas, 2011). Y es aquí donde la comunicación podría desempeñar un papel interesante a la vez que necesario, ante la necesidad de empoderar a colectivos similares (Moriel-Corral, Pizarro, Hernández-Hernández y Bonilla-Loyo, 2012). Emocionar hace referencia al conjunto de interacciones entre los factores subjetivos y objetivos, que son mediados por sistemas neurales y hormonales, generando sentimientos, procesos cognitivos, ajustes fisiológicos y que generan una conducta frecuentemente expresiva cuya finalidad es la adaptación (Kleinginna y Kleinginna, 1981). Nos preguntamos, ¿Qué sería del cine si no fuera un vehículo de emociones? ¿Qué sería de un doctor si no entendiera las emociones y necesidades de un paciente? Para tratar de comprender el concepto de emoción consideramos oportuno recurrir a su definición etimológica, ya que se trata de un concepto en sí mismo difícil de acotar por su multidimensionalidad y perspectiva (Barrientos, 2019). Así descubrimos que es una palabra proveniente del latín e+movere, y que su traducción al castellano podría ser interpretada como "lo que nos empuja o mueve a". En el caso del constructo emoción, encontramos 
Necesidad de formar a los cuidadores de personas en situación de dependencia:

comunicación y respuesta desde la UCM

consenso a nivel científico sobre su definición. Mayoritariamente se considera que una emoción es "un estado complejo del organismo caracterizado por una excitación o perturbación que predispone a una respuesta organizada" (Bisquerra, 2003, p. 49). La definición que aporta la RAE para este vocablo es muy similar describiéndola como "una alteración del ánimo intensa y pasajera, agradable o penosa, que va acompañada de cierta conmoción somática". Por tanto, se refiere a un proceso conductual del cual deriva una alteración física asociada. Es importante por tanto empatizar con la persona a la que se cuida a través del control y la gestión de las emociones de nosotros mismos y las de los demás. De esta forma resulta más fácil comunicarnos con claridad, sentimiento y profesionalidad. El vínculo que genera la correcta comunicación con el paciente o "cuidado" es fundamental para el buen desarrollo y bienestar de la persona que necesita atenciones básicas sintiendo el "calor humano" del cuidador, porque es con él/ella con quien pasará largas horas del día, largas horas de la noche y por tanto emergerán emociones de diversa índole, necesarias todas, para contemplar el día a día con cierto grado de optimismo y satisfacción de un trabajo bien realizado. Paul Watzlawich compartimentó la comunicación humana en cinco secciones ineludibles y dejó escrito en Teoría de la comunicación humana (1997), actuar de cualquier modo implica necesaria e inevitablemente transmitir información a nuestro entorno humano: en otras palabras, no es posible no comunicar, pues hasta la decisión de no comunicarnos transmite información: como mínimo, la información de que hemos cesado de comunicarnos. Para Watzlavich comunicar es tomar partido y definirse de cara a las relaciones personales. Si trasladamos sus palabras a nuestro caso de estudio podemos comprender que comunicar es transmitir, y los cuidadores sólo deben transmitir positividad y optimismo al "cuidado" porque es en ellos en quienes confían, es en ellos en quienes dejan la responsabilidad de ser cuidados y protegidos. Adelantamos pues, una conclusión cuanto menos importante, y es que la comunicación con emoción puede representar una guía de valores y comportamientos para las personas que precisan ser cuidadas.

La figura del "cuidador no profesional" es tan novedosa y tan escasamente conocida que hay incluso discrepancias sobre su denominación. Para algunos autores deben ser considerados como "cuidadores no profesionales", distinguiéndolos claramente de los enfermeros que son cuidadores profesionales. Para otros la figura se denomina claramente "cuidadores informales» e incluso hay una tercera corriente denominativa que se decanta por la "cuidadores familiares" (Como se desprende igualmente del trabajo de Ruíz López, Pullas Tapia, Parra Parra y Zamora Sánchez, 2017) y aun una cuarta que los califica como "cuidadores primarios". Sin embargo, nos parece más acertada la denominación de "cuidadores principales" o simplemente la de "cuidadores", alejándola de partículas semánticas invalidantes como "no" e "in" que tienden a deteriorar la figura o "familiar" o "primario" que pueden ocasionar cierta confusión terminológica.

\subsection{Perspectivas demográficas}

En España, según las proyecciones de población entre 2016 y 2066 se espera una pérdida de población de algo más de medio millón de habitantes en los próximos quince años y 5,4 millones hasta 2066. Con respecto al porcentaje de población de 65 años y

Revista de Comunicación y Salud, 2019, Vol. 9, nº 1, pp. 19-38 
Necesidad de formar a los cuidadores de personas en situación de dependencia:

comunicación y respuesta desde la UCM

más que actualmente se sitúa en el 18,7\%, alcanzaría el $25,6 \%$ en 2031 y el $34,6 \%$ en 2066.

Por otro lado, la esperanza de vida al nacimiento alcanzaría los 83,2 años en los varones y los 87,7 en las mujeres en 2031 , lo que supone una ganancia respecto a los valores actuales de 3,3 y de 2,3 años, respectivamente.

En 2065, de mantenerse la tendencia actual, la esperanza de vida de los hombres superaría los 88,5 años y la de las mujeres los 91,6 años. De la misma forma, una mujer que alcanzase la edad de 65 años en 2065 viviría en promedio otros 28,2 años más (25,1 en los hombres), frente a los 22,7 años de supervivencia actuales (18,8 para los hombres). (INE, 2016, p. 3).

\section{Proyección de la esperanza de vida al} nacimiento y a los 65 años.

\begin{tabular}{|c|c|c|c|c|}
\hline \multirow[t]{2}{*}{ Años } & \multicolumn{2}{|c|}{$\begin{array}{l}\text { Esperanza de vida al } \\
\text { nacimiento }\end{array}$} & \multicolumn{2}{|c|}{$\begin{array}{l}\text { Esperanza de vida a los } \\
65 \text { años }\end{array}$} \\
\hline & Hombres & Mujeres & Hombres & Mujeres \\
\hline 2015 & 79,94 & 85,41 & 18,81 & 22,67 \\
\hline 2016 & 80,26 & 85,71 & 19,08 & 22,97 \\
\hline 2021 & 81,31 & 86,41 & 19,72 & 23,55 \\
\hline 2026 & 82,30 & 87,08 & 20,37 & 24,13 \\
\hline 2031 & 83,23 & 87,74 & 21,01 & 24,70 \\
\hline 2036 & 84,12 & 88,37 & 21,64 & 25,26 \\
\hline 2041 & 84,97 & 88,98 & 22,27 & 25,80 \\
\hline 2046 & 85,79 & 89,57 & 22,89 & 26,34 \\
\hline 2051 & 86,57 & 90,14 & 23,50 & 26,86 \\
\hline 2056 & 87,32 & 90,69 & 24,09 & 27,37 \\
\hline 2061 & 88,04 & 91,22 & 24,68 & 27,86 \\
\hline 2065 & 88,60 & 91,64 & 25,13 & 28,25 \\
\hline
\end{tabular}

\section{Figura 6}

A pesar de la pérdida de población y la mayor esperanza de vida, el número de defunciones seguiría creciendo como consecuencia del envejecimiento poblacional. Así, en el periodo $2016-2030$ se llegarían a registrar casi seis millones y medio de defunciones, un $12,7 \%$ más que las observadas en los 15 años previos (2001-2015) (INE, 2016, p. 5).

(...) la población se incrementaría en la mitad superior de la pirámide de población. De hecho, todos los grupos de edad a partir de los 70 años experimentarían un crecimiento de efectivos. Dentro de 15 años en España residirían 11,7 millones de personas mayores de 64 años, tres millones más que en la actualidad (un $34,8 \%$ ). Y esta cifra se incrementaría hasta 14,2 millones de personas (un 63,1\% más) en 50 años. Si observamos los grupos de edad quinquenales, el más numeroso en la actualidad es el de 40 a 44 años. Pero esto cambiará tanto en 2031 como en 2066, cuando el grupo con más efectivos sería el de 55 a 59 años. (INE, 2016, p. 7). 
Necesidad de formar a los cuidadores de personas en situación de dependencia: comunicación y respuesta desde la UCM

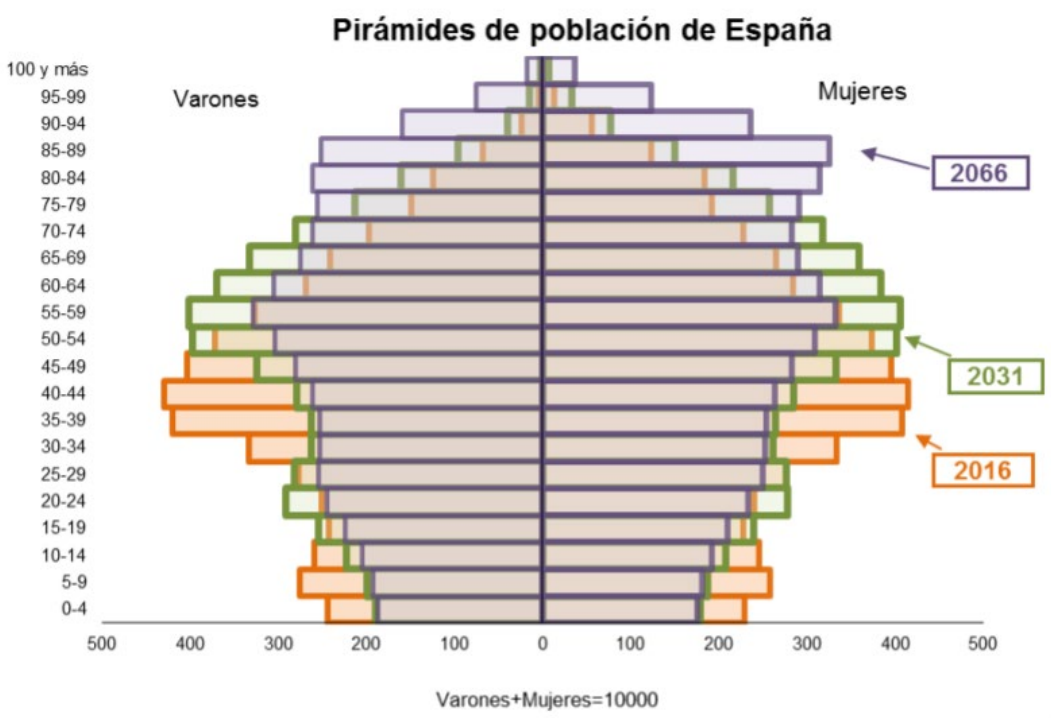

Figura 7

Envejecimiento demográfico. El porcentaje de población de 65 años y más, que actualmente se sitúa en el $18,7 \%$ del total de la población, pasaría a ser del $25,6 \%$ en 2031 , y del $34,6 \%$ en 2066 . De mantenerse las tendencias actuales, la tasa de dependencia (el cociente, en tanto por ciento, entre la población menor de 16 años o mayor de 64 y la población de 16 a 64 años) se elevaría desde el $53,5 \%$ actual hasta el $62,2 \%$ en 2031 , alcanzando el $87,7 \%$ en 2066 . La población centenaria (los que tienen 100 años o más) pasaría de las 16.460 personas en la actualidad a más de 222.104 dentro de 50 años. (INE, 2016, p. 8).

\section{PROYECTO “CUIDADOR COMPLUTENSE”}

Desde la Facultad de Enfermería, Fisioterapia y Podología de la Universidad Complutense de Madrid (FEF y P-UCM) consideramos estas necesidades formativas de los cuidadores como propias y tratamos de liderar un proceso formativo para ellos, que garantice una atención de calidad y que por lo tanto incluya las condiciones de eficiencia y seguridad en el cuidado, hacia las personas con algún grado de dependencia temporal o permanente que están cuidando.

\subsection{Objetivos del Proyecto "Cuidador Complutense"}

Una vez analizada la situación desde un punto de vista demográfico y sociológico, que retrata la perspectiva de una sociedad española envejecida, y que anuncia un progresivo envejecimiento y en consecuencia, un incremento de la población mayor y de la dependencia y la vulnerabilidad, este Proyecto se plantea los siguientes objetivos:

1. Promover la cultura del autocuidado y del heterocuidado en la población.

2. Acercar las técnicas y procedimientos básicos del cuidado a la población en general y a los cuidadores y alumnos enfermeros en particular. 
Necesidad de formar a los cuidadores de personas en situación de dependencia:

comunicación y respuesta desde la UCM

3. Liderar y promover tanto el autocuidado como el heterocuidado en la población en general, los cuidadores y los alumnos enfermeros.

\subsection{Destinatarios inmediatos de este Proyecto "Cuidador complutense"}

- La población en general interesada en el autocuidado como en el heterocuidado.

- Los cuidadores.

- Los alumnos actuales y posibles de Enfermería.

Todos ellos, colectivos con interés en el autocuidado y en el heterocuidado y con habilidades tecnológicas suficientes como para acceder a los contenidos desde cualquier terminal tecnológico.

\subsection{Destinatarios finales de este Proyecto «Cuidador Complutense»:}

Personas que debido a:

- Un deterioro agudo de su salud, precisan apoyo en sus cuidados.

- Un deterioro crónico de su salud, precisan cuidados permanentes.

- Una situación de dependencia, precisan de un cuidador principal permanente.

- Población en general que quiera conocer o mejorar sus habilidades en el autocuidado de su salud.

\section{METODOLOGÍA Y PLAN DE TRABAJO}

Se pretende aplicar un método participativo para que los alumnos logren sensibilizarse ante la tarea del cuidado que deben realizar. Desde la FEF y P-UCM contribuiremos al diseño y mantenimiento de aquellos recursos informativos y didácticos, tanto presenciales como a distancia, con los que nuestros alumnos se encuentren más identificados: instauración de clases presenciales, realización de prácticas con modelos de simulación, diseño y mantenimiento de la página web, de las redes sociales, grabación de entrevistas y vídeos y diseño de material tangible.

\subsection{Sobre los destinatarios universitarios}

Con este proyecto, los alumnos y también los profesores de la FEF y P-UCM, pero también los de otras Facultades de la UCM podrán desde una perspectiva de Aprendizaje Servicio (APS):

- Realizar un inestimable servicio a los cuidadores, tanto en el ámbito español como internacional, ya que los recursos digitales que se utilizan tienen un componente divulgativo que traspasa nuestras fronteras.

- Aprender y divulgar las técnicas del cuidado entre los cuidadores, así como entre la población en general.

- En el caso concreto de los alumnos de la FEF y P-UCM, podrán aplicar y consolidar los conocimientos aprendidos en clase, así como las habilidades y actitudes aprendidas en sus prácticas sanitarias y utilizarlos en la vida real como forma de ayuda a los cuidadores y a la población en general.

Revista de Comunicación y Salud, 2019, Vol. 9, n 1, pp. 19-38 
Necesidad de formar a los cuidadores de personas en situación de dependencia: comunicación y respuesta desde la UCM

- Los alumnos de las Ciencias de la Salud en general: Psicología, Farmacia, Medicina, etc. e incluso de otras Facultades alejados de la rama sanitaria también podrán acceder a un nuevo aspecto de la persona en situación de vulnerabilidad y dependencia desde una perspectiva del cuidado y la comunicación, ofreciendo una respuesta solidaria.

- Todos los alumnos de la UCM podrán comprometerse con una situación de déficit en el ámbito del conocimiento, desarrollando un proyecto solidario y enormemente útil.

- Así mismo podrán percibir la importancia de los valores: empatía, ética, solidaridad y comprensión en la educación y en la sociedad.

- También podrán fomentar su autonomía personal, resolviendo problemas y tomando decisiones de manera frecuente de una manera responsable y eficiente, así como concienciarse sobre la importancia de sus conocimientos y de la necesidad de divulgarlos a una población que carece de ellos, demostrando así la utilidad de sus aportaciones.

Con este Proyecto, tanto la FEF y P-UCM como la UCM en general, obtendrán un reconocimiento de la sociedad ya que una institución educativa pública como la UCM se compromete a divulgar las mejores prácticas del cuidado, científicamente estudiadas en su seno a la población en general, lo que contribuye a mejorar su imagen pública. Reflejando el pensamiento de Ortega y Gasset cuando nos hablaba de la «Misión de la Universidad», podemos concretar que la modalidad de Aprendizaje-Servicio permite responder a la pregunta que se hacía el autor de una manera eficiente, ya que la Universidad pública se convierte así en promotora de la cultura y del pensamiento crítico alejándonos de la fragmentación del saber académico, y como también refieren Santos Rego, M.A.; Sotelino Losada, A. y Lorenzo Moledo:

Hace ya tres lustros, uno de los autores de este libro hizo una visita académica a la Georgetown University en Washington, D.C. Allí, en la magnífica biblioteca de este renombrado centro de educación superior, tuvo ocasión de consultar una obra de sugerente título, A passion for democracy, cuyo autor es Benjamin R. Barber (1998). Y leyó algo que siempre habría de recordar: no es que la universidad tenga una misión cívica, es civilidad en sí misma, que se define a través de las reglas y convenciones que facilita el diálogo en una comunidad y la clase de discurso del que todo conocimiento depende. (Santos Rego, M. A.; Sotelino Losada, A. y Lorenzo Moledo, M., 2015, p. 7).

\subsection{Recursos}

\begin{tabular}{|c|c|}
\hline \multicolumn{2}{|r|}{ Recursos } \\
\hline $\begin{array}{l}\text { 30. } \\
\text { Cestión de páginas Web }\end{array}$ & $\begin{array}{l}\text { PÁGINA WEB: centraliza el Proyecto. Incluye enlaces al resto de los } \\
\text { recursos. Los profesores pueden redactar entradas, incluir recursos } \\
\text { audiovisuales y prescribir recomendaciones. } \\
\text { https://www.ucm.es/admin/apps/?apn=web }\end{array}$ \\
\hline 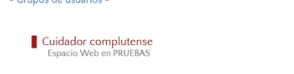 & \\
\hline
\end{tabular}


Necesidad de formar a los cuidadores de personas en situación de dependencia: comunicación y respuesta desde la UCM

\begin{tabular}{|c|c|}
\hline 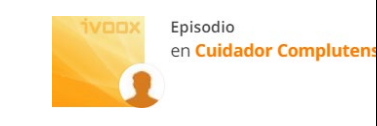 & $\begin{array}{l}\text { CANAL DE AUDIO EN IVOOX: grabación de entrevistas breves } \\
\text { (tres minutos) a los profesores en formato podcast. } \\
\text { https://www.ivoox.com/cuidador-complutense sb.html? }\end{array}$ \\
\hline & $\begin{array}{l}\text { App: Realizada por el Departamento de Arquitectura de } \\
\text { Computadores y Automática, Facultad de Informática de la UCM. } \\
\text { Modalidad a distancia: EAO. }\end{array}$ \\
\hline 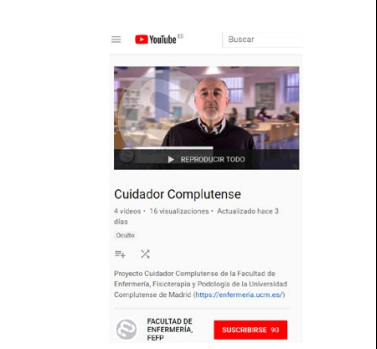 & $\begin{array}{l}\text { CANAL DE víDEO corporativo (UCM) en YOUTUBE } \\
\text { Incluye documentación complementaria. } \\
\text { https://www.youtube.com/playlist?list=PLIpARNby2vcXLAMJBILAO0 } \\
\underline{\text { XBhWJOPc4fB }}\end{array}$ \\
\hline Eutbear'y & TWITTER: @CuidadorC \\
\hline (1) & $\begin{array}{l}\text { INSTAGRAM } \\
\text { https://www.instagram.com/complutensecuidador/?hl=es }\end{array}$ \\
\hline ffrow & $\begin{array}{l}\text { FACEBOOK } \\
\text { https://www.facebook.com/complutense.cuidador.3 }\end{array}$ \\
\hline (G) WhatsApp & Cuidador Complutense \\
\hline $\begin{array}{l}\text { UNIVERSIDA } \\
\text { COMPLUTENS } \\
\text {. shop }\end{array}$ & $\begin{array}{l}\text { Tienda Complutense: edición de calendarios, agendas, carteles y } \\
\text { juegos didácticos para cuidadores. }\end{array}$ \\
\hline
\end{tabular}

\section{IMPACTO, VIABILIDAD, ADECUACIÓN Y TRANSFERENCIA DEL PROYECTO "CUIDADOR COMPLUTENSE"}

Este Proyecto formativo trata de responder desde la calidad docente a las necesidades de formación inicial y permanente de los cuidadores en torno a dos perspectivas, una de ellas legislativa y otra educativa, que pueden actuar de manera sinérgica:

1. La Ley $39 / 2006$ de 14 de diciembre, de promoción personal de la autonomía personal y atención a las personas en situación de dependencia.

2. La propuesta metodológica de Aprendizaje-Servicio de la UCM.

\subsection{Perspectiva legislativa}

Por una parte, La Ley 39/2006 de 14 de diciembre, de promoción personal de la autonomía personal y atención a las personas en situación de dependencia refiere en su EXPOSICIÓN DE MOTIVOS, entre otras cosas lo siguiente: 
Necesidad de formar a los cuidadores de personas en situación de dependencia: comunicación y respuesta desde la UCM

1. La atención a las personas en situación de dependencia y la promoción de su autonomía personal constituye uno de los principales retos de la política social de los países desarrollados. El reto no es otro que atender las necesidades de aquellas personas que, por encontrarse en situación de especial vulnerabilidad, requieren apoyos para desarrollar las actividades esenciales de la vida diaria, alcanzar una mayor autonomía personal y poder ejercer plenamente sus derechos de ciudadanía.

3. La atención a este colectivo de población se convierte, pues, en un reto ineludible para los poderes públicos, que requiere una respuesta firme, sostenida y adaptada al actual modelo de nuestra sociedad. No hay que olvidar que, hasta ahora, han sido las familias, y en especial las mujeres, las que tradicionalmente han asumido el cuidado de las personas dependientes, constituyendo lo que ha dado en llamarse el "apoyo informal". Los cambios en el modelo de familia y la incorporación progresiva de casi tres millones de mujeres, en la última década, al mercado de trabajo introducen nuevos factores en esta situación que hacen imprescindible una revisión del sistema tradicional de atención para asegurar una adecuada capacidad de prestación de cuidados a aquellas personas que los necesitan.

Y concretamente en el CAPÍTULO II, Sección $2^{\mathrm{a}}$, refiere la importancia de la formación para el colectivo de los cuidadores no profesionales:

Artículo 18; El Consejo Territorial del Sistema para la Autonomía y Atención a la Dependencia promoverá acciones de apoyo a los cuidadores no profesionales que incorporarán programas de formación, información y medidas para atender los periodos de descanso.

Artículo 36. Formación y cualificación de profesionales y cuidadores.

1. Se atenderá a la formación básica y permanente de los profesionales y cuidadores que atiendan a las personas en situación de dependencia. Para ello, los poderes públicos determinarán las cualificaciones profesionales idóneas para el ejercicio de las funciones que se correspondan con el Catálogo de servicios regulado en el artículo 15.

2. Los poderes públicos promoverán los programas y las acciones formativas que sean necesarios para la implantación de los servicios que establece la Ley.

3. Con el objetivo de garantizar la calidad del Sistema, se fomentará la colaboración entre las distintas Administraciones Públicas competentes en materia educativa, sanitaria, laboral y de asuntos sociales, así como de éstas con las universidades, sociedades científicas y organizaciones profesionales y sindicales, patronales y del tercer sector.

\subsection{Perspectiva educativa}

En la Convocatoria Proyectos Aprendizaje-Servicio Complutense 2019 se refiere:

1.2. En esta misma línea, la Universidad Complutense está comprometida con la incorporación de metodologías innovadoras que permita vincular nuestra institución con la realidad socioeconómica de su entorno, facilitando que sus

Revista de Comunicación y Salud, 2019, Vol. 9, n 1, pp. 19-38 
Necesidad de formar a los cuidadores de personas en situación de dependencia: comunicación y respuesta desde la UCM

estudiantes desarrollen competencias gracias a través de la participación en la generación de beneficios para la comunidad en la cual se inscribe la Complutense.

1.3. La metodología conocida como "Aprendizaje-Servicio" (en adelante, ApS), permite abordar dichos objetivos de manera eficaz y adecuada, vinculando a estudiantes y profesorado con proyectos de especial relevancia social. De hecho, la metodología ApS ha venido ganando presencia en diversas materias de los planes de estudios de la Universidad, así como en las propuestas de grupos de trabajo en el ámbito de la innovación educativa.

Por lo tanto, nuestro Proyecto se enmarca claramente en estas premisas, tanto desde el punto de vista de aprendizaje como de servicio permanente a la sociedad.

\subsection{Divulgación y promoción del Proyecto}

En este caso, la transferencia del Proyecto pretende hacer llegar al colectivo heterogéneo y disperso de cuidadores, mediante diversos canales digitales de fácil acceso, la información e información necesaria, impartida por los profesionales de la FEF y P-UCM para que realicen su importante labor de cuidado con mejores garantías.

El proyecto "Cuidador complutense" pretende la promoción del proyecto en los medios de comunicación y redes sociales mediante su divulgación en:

- La Facultad de Enfermería, Fisioterapia y Podología (FEF y P).

- La Universidad Complutense de Madrid.

- Otras Facultades de otras Universidades nacionales y extranjeras.

- Instituciones corporativas enfermeras: Colegios profesionales enfermeros y Consejo General de Enfermería.

- Las Asociaciones de enfermos.

- Las Embajadas de países latinoamericanos en España.

- Los Ministerios de Educación y Sanidad de países latinoamericanos.

\subsection{Proyección futura del Proyecto}

Una vez establecidos los recursos didácticos previstos y garantizada su continuidad, el alumno podrá realizar gratuitamente el curso "Cuidador Complutense" en formato APP según su propio ritmo de aprendizaje. De acuerdo con la normativa de los cursos de Formación Continua de la UCM se facilitará la realización de un examen presencial en la FEF y P-UCM mediante el abono de una tasa mínima, que tras ser aprobado garantice la expedición del correspondiente certificado acreditativo.

Dejaremos par más adelante otras muchas posibilidades previstas de este Proyecto, tales como:

- La creación y gestión de una Bolsa de empleo para cuidadores. 
Necesidad de formar a los cuidadores de personas en situación de dependencia: comunicación y respuesta desde la UCM

- La propuesta de diseños de investigación enfermera en torno a esta actividad de Aprendizaje-Servicio enmarcadas, bien en Trabajos Fin de Grado, Trabajos Fin de Máster o en Doctorados.

- La publicación de recursos didácticos fruto de la experiencia acaecida.

- La divulgación de los contenidos del Proyecto en los medios de comunicación.

\subsection{Transferencia y adecuación}

Woolfolk (2010, p. 295-296) recopila el concepto de transferencia con respecto a los trabajos de cuatro autores, especificando dos tipos principales:

De acuerdo con Salomon y Perkins (1989, p. 118) podemos establecer dos tipos de transferencia, de bajo y alto nivel. La primera supone la transferencia espontánea y automática de habilidades que requieren mucha práctica, pero con poca necesidad de pensamiento reflexivo, mientras que la segunda, la de alto nivel, requiérela aplicación consciente de conocimientos abstractos, aprendidos en una situación a otra situación diferente.

Bransford y Schwartz (1999) consideran que es este tipo de transferencia de alto nivel el que ofrece una mejor preparación para el aprendizaje futuro, ya que la clave para la transferencia de alto nivel es la abstracción consciente o la identificación deliberada de un principio, idea principal, estrategia o procedimiento que no esté vinculado con un problema o situación específicos, sino que se aplique a muchos casos. Este tipo de abstracción se vuelve parte de su conocimiento metacognoscitivo que está disponible para guiar el aprendizaje y la resolución de problemas futuros. Bransford y Schwartz (1999) añadieron otra clave: un ambiente rico en recursos que apoye una transferencia productiva y apropiada.

En este Proyecto se facilitan estos dos tipos de transferencia, aunque priorizando la de alto nivel ya que:

Se identifica el problema: la falta de información y formación de los cuidadores en al ámbito doméstico, donde suelen desarrollar sus tareas, alejados del sistema educativo universitario.

Se busca y ofrecen soluciones eficientes para el cuidado, desde el propio sistema educativo universitario que pueden ser aplicadas de manera idónea en dicho ámbito doméstico con suficientes garantías.

\subsection{Viabilidad y alcance del Proyecto "Cuidador Complutense"}

Análisis de la situación: realizada en el primer punto de la descripción de este Proyecto, podemos resumirla de esta manera:

Recientemente es posible detectar un segmento creciente de la población española en situación de vulnerabilidad y dependencia fruto de los progresos científicos en el 
Necesidad de formar a los cuidadores de personas en situación de dependencia:

comunicación y respuesta desde la UCM

ámbito de la salud y de las nuevas situaciones sociológicas y demográficas, que es atendida por cuidadores con graves carencias formativas.

Alcance del Proyecto. En consecuencia, con este proyecto "Cuidador Complutense" se pretende ofrecer recursos didácticos enfermeros eficientes de libre acceso y divulgación, sobre el cuidado a personas con vulnerabilidad y dependencia, así como configurar un corpus de información y conocimiento enfermero que contribuya a la mejora eficiente del cuidado por parte de los cuidadores.

\section{REFERENCIAS}

Artaza, I. y Ramos, P. (2018). Estudio de investigación sociosanitaria sobre cuidadores de personas mayores dependientes. Sociedad Española de gerontología, Fundación Envejecimiento y Salud. Madrid: LINDOR.

Barrientos, A. (2019). Tesis Doctoral: El desarrollo de la Inteligencia Emocional en los estudios del Grado en Turismo en la Universidad de La Laguna (Tenerife). Universidad Camilo José Cela. Madrid.

Bisquerra, R. (2003). Educación emocional y competencias básicas para la vida. Revista de Investigación Educativa, 21(1), 7-43.

BOE $n^{\circ} 299$, de 15/12/2006. Promoción de la Autonomía Personal y Atención a las personas en situación de dependencia favoreciendo su desarrollo. Recuperado de https://www.boe.es/eli/es///2006/12/14/39/con

Bransford, J. \& Schwartz, D. (1999). Rethinking transfer: A simple proposal with multiples implications. En A. Iran-Nejad y P. D. Pearson (eds.), Review of research in education, 24, 61-100.

Encuesta Nacional de Salud (2017). Recuperado de

https://www.ine.es/dyngs/INEbase/es/operacion.htm?c=Estadistica C\&cid=12547361 $\underline{76783 \& \text { menu}=\text { resultados\&idp }=1254735573175}$

Encuesta Nacional de Salud. (2017). Nota Técnica. Ministerio de Sanidad, Consumo y Bienestar Social. Gobierno de España. Recuperado de https://www.mscbs.gob.es/estadEstudios/estadisticas/encuestaNacional/encuestaNa c2017/ENSE2017 notatecnica.pdf

Informe Mundial sobre la Discapacidad (2011). Recuperado de https://www.who.int/disabilities/world report/2011/summary es.pdf?ua=1

Instituto Nacional de Estadística (2016). Proyecciones de población. Recuperado de https://www.ine.es/prensa/np994.pdf 
Necesidad de formar a los cuidadores de personas en situación de dependencia: comunicación y respuesta desde la UCM

Kleinginna, P. \& Kleinginna, A. (1981). A categorized list of emotion definitions, with suggestions for a consensual definition. Motivation and emotion, 5(4), 345-379.

Martínez Pérez, A. (2010). El síndrome Burnout. Evolución conceptual y estado actual de la cuestión. Revista de Comunicación Vivat Academia, Año XIII, 112, 42-80. doi: http://dx.doi.org/10.15178/va.2010.112.42-80

Ministerio de Sanidad, Consumo y Bienestar Social. Gobierno de España. Recuperado de https://www.mscbs.gob.es/estadEstudios/estadisticas/encuestaNacional/encuestaNa c2017/ENSE2017 notatecnica.pdf

Moriel-Corral, L., Pizarro, N., Hernández-Hernández, H. y Bonilla-Loyo, E. (2012). Empoderamiento de enfermería como profesión y sujeto social: caso chihuahua. Revista de Comunicación de la SEECl, XVI(2), 30-38. doi: http://dx.doi.org/10.15198/seeci.2012.27.30-38

Rivas, J. y Ostiguín, R. (2011). Cuidador: ¿concepto operativo o preludio teórico? Revista Enfermería Universitaria ENEO-UNAM, 8(8), 1.

Ruíz López, P., Pullas Tapia, P., Parra Parra, C.A. y Zamora Sánchez, R. (2017). La doble presencia en las trabajadoras femeninas: equilibrio entre el trabajo y la vida familiar. Revista de Comunicación de la SEECI, XXI(44), 33-51. doi: http://dx.doi.org/10.15198/seeci.2017.44.33-51

Salomon, G. y Perkins, D. (1989). Rocky roads to transfer: Re-thinking mechanisms of a neglected phenomenon. Educational Psychologist, 24, 113-142.

Santos, M., Sotelino, A. y Lorenzo, M. (2015). Aprendizaje-servicio y misión cívica de la universidad Una propuesta de desarrollo. Barcelona: Octaedro.

Suberviola-Ovejas, I. (2011) Competencia emocional y rendimiento académico en el alumnado universitario. Revista de Comunicación Vivat Academia, 117E, 1-17. doi: http://dx.doi.org/10.15178/va.2011.117E.1-17

Watzlawick, P., Beavin, J. y Jackson, D. (1997). Teoría de la comunicación humana. Barcelona: Herder.

Woolfolk, A. (2010). Psicología educativa. México: Pearson.

Revista de Comunicación y Salud, 2019, Vol. 9, nº 1, pp. 19-38 